\title{
Relaciones de pareja como factor relacionado con la práctica del autoexamen de mama en estudiantes universitarias
}

\author{
Couple relationships as a factor related to the practice of breast self-examination in university students
}

\author{
Anaid Guadalupe Martín-Díaz' orcid.org/0000-0002-0507-3132 \\ Yolanda Campos-Uscanga ${ }^{2}$ orcid.org/0000-0002-51 14-3621 \\ Claudia Gutiérrez Sida ${ }^{3 *}$ orcid.org/0000-0002-5349-3326
}

1 Universidad Veracruzana. Veracruz, México

2 Instituto de Salud Pública, Universidad Veracruzana. Veracruz, México

3 Facultad de Psicología, Universidad Veracruzana. Veracruz, México

Martín-Díaz AG, Campos-Uscanga Y, Gutiérrez-Sida C. Relaciones de pareja como factor relacionado con la práctica del autoexamen de mama en estudiantes universitarias. Univ. Salud. 2018;20(3):227-235. DOI: http://dx.doi.org/10.22267/rus.182003.125

\begin{abstract}
Resumen
Introducción: Existe controversia sobre la relación entre las creencias y la realización de la autoexploración mamaria en las mujeres y se carece de estudios que muestren la influencia que las parejas pueden tener en dicha práctica. Objetivo: Identificar si existe asociación entre el autoexamen de mama, los estilos de vida, las creencias en salud y las relaciones de pareja en estudiantes universitarias. Materiales y métodos: Estudio transversal con 284 mujeres estudiantes de la Universidad Veracruzana. Se aplicó el cuestionario de Creencias en salud hacia el autoexamen de mama y un cuestionario de datos personales. Resultados: La media de edad fue 20,32 años. Solo $19,7 \%$ de las estudiantes reportaron realizar el autoexamen de mama de manera mensual. Las mujeres que no tenían pareja mostraron mayores barreras hacia el autoexamen y menores beneficios percibidos. La edad favoreció la realización del autoexamen de mama, mientras que las barreras percibidas lo limitaron. Por su parte, tener pareja se asoció con menores barreras y mayores beneficios percibidos hacia el autoexamen. Conclusiones: Existe baja realización del autoexamen en las estudiantes a pesar del conocimiento que poseen del mismo. Tener pareja disminuye las barreras percibidas hacia la realización del autoexamen de mama.
\end{abstract}

Palabras clave: Autoexamen de mamas; prevención primaria; conocimientos, actitudes y práctica en salud; estilo de vida; autocuidado. (Fuente: DeCS, Bireme).

\begin{abstract}
Introduction: There is controversy about the relationship between beliefs and the realization of breast selfexamination in women and there is a lack of studies which show the influence that couples can have in that practice. Objective: To identify whether there is an association between breast self-examination, lifestyles, health beliefs, and partner relationships in university students. Materials and methods: A cross-sectional study was conducted with 284 women students from the University of Veracruz. The Health beliefs questionnaire was applied to breast selfexamination and a personal data questionnaire. Results: The average age was 20.32 . Only $19.7 \%$ of the students reported the realization of breast self-examination monthly. Women who did not have a couple showed greater barriers to self-examination and lower perceived benefits. Age favored the realization of breast self-examination, while the perceived barriers limited it. On the other hand, having a couple was associated with lower barriers and increased benefits to self-examination. Conclusions: There is low self-examination in the students despite their knowledge. Having a couple decreases the perceived barriers to conducting breast self-examination.
\end{abstract}

*Autor de correspondencia

Claudia Gutiérrez Sida

e-mail: cguiettrez@uv.mx 
Key words: Breast self-examination; primary prevention; health knowledge, attitudes, practice; life style; self-care. (Source: DeCS, Bireme).

\section{Introducción}

En América Latina, la incidencia de cáncer de mama se ha incrementado debido al envejecimiento poblacional, los patrones reproductivos, mayor exposición a factores de riesgo, la modificación de estilos de vida, así como los problemas de acceso oportuno a la detección y el diagnóstico temprano(1). La mortalidad por cáncer de mama es más elevada en los países en desarrollo, ya que generalmente el diagnóstico se realiza en fases avanzadas de la enfermedad, debido a la falta de acceso a servicios de salud y a la poca sensibilización para la detección precoz ${ }^{(2)}$.

En México, el cáncer de mama constituye una causa importante de morbilidad y mortalidad, sin embargo, y aunque el autoexamen de mama es una estrategia fundamental en la prevención de este problema de salud su aplicación es limitada(3). Existe evidencia de que en países en desarrollo con elevada mortalidad por esta enfermedad, el autoexamen debería ser una de las medidas más empleadas por la limitación de recursos para realizar cribados(4). El autoexamen ocupa un lugar preferente dentro del marco de posibilidades de diagnóstico precoz de esa patología, dado que permite familiarizar a la mujer con la forma, volumen, coloración, movilidad, consistencia y sensibilidad de ambas mamas y poder detectar cualquier cambio para reportarlo a su médico lo antes posible(5).

Son pocos los estudios que revisan aspectos cognitivos y su relación con la adopción de conductas de autocuidado para la detección de enfermedades crónicas como el cáncer de mama en la población estudiantil. De los modelos teóricos que en el ámbito de la psicología de la salud se han propuesto para intentar explicar la ocurrencia de conductas saludables y preventivas de la enfermedad, el modelo de creencias de salud de Becker ha sido el que más atención ha suscitado y el que ha generado mayor número de estudios(6). Surge como propuesta para explicar y predecir el cambio o mantenimiento del comportamiento preventivo, resultado de la interacción de ciertas creencias de las personas, utilizado en materia de promoción y educación para la salud(7).

Algunas creencias en salud asociadas a la realización de la autoexploración son, la susceptibilidad y gravedad percibida hacia el cáncer de mama, la percepción de beneficios y las barreras percibidas hacia el autoexamen $(8,9)$. En otros estudios se ha identificado que la falta de conocimiento, considerarse sin riesgo de padecer cáncer de mama, la ausencia de asesoramiento médico(10,11) y no tener antecedentes familiares para cáncer de mama(11) se asociaron con las barreras percibidas hacia el autoexamen de mama. También existe evidencia de que la práctica del autoexamen se relaciona con factores sociodemográficos(12) entre ellos la edad(9).

Aunque existe evidencia de una posible relación entre las creencias y la realización de la autoexploración mamaria en las mujeres, se carece de estudios que muestren la asociación que el tener relaciones de pareja como noviazgo, pueden tener en la realización de la autoexploración y a su vez si la presencia de pareja se relaciona con las creencias en salud. Lo anterior cobra especial relevancia en grupos de mujeres jóvenes, que si bien no están casadas, tienen relaciones de pareja que pudieran ser determinantes para el cuidado de la salud. Lo anterior es importante considerando que en mujeres casadas se ha observado mayor predisposición a la práctica del autoexamen en comparación con las solteras $(10,12)$, pero estos análisis no han considerado si estas mujeres solteras tienen o no relaciones de pareja. Por lo anterior el objetivo de esta investigación fue identificar si existe relación entre la práctica del autoexamen de mama, las creencias en salud, los estilos de vida y las relaciones de pareja en mujeres estudiantes universitarias. 


\section{Materiales y métodos}

\section{Muestra}

Se realizó un estudio transversal y analítico. Para el cálculo de la muestra se utilizó la fórmula para estimar una proporción con población finita, considerando un intervalo de confianza de 95\%, teniendo como referencia una población de 1.786 estudiantes pertenecientes a las seis áreas académicas (ciencias de la salud, artes, ciencias biológicas- agropecuarias, económicoadministrativas, humanidades) de la Universidad Veracruzana, región Xalapa, Veracruz, México, del primer a octavo semestre inscritas en el periodo agosto diciembre 2016. Con una prevalencia esperada de realización del autoexamen de mama en mujeres de $28 \%{ }^{(11)}$ y un nivel de confianza de $95 \%$, se obtuvo un tamaño de muestra de 284 estudiantes universitarias. El periodo de recolección de datos fue de 2 meses, durante el periodo agosto diciembre 2016.

\section{Instrumentos}

Se aplicó un cuestionario con datos sociodemográficos, el cual incluyó edad, lugar de nacimiento, presencia de pareja (noviazgo), grado educativo y semestre cursado, presencia de trabajo, derechohabiencia y antecedentes heredofamiliares por cáncer de mama.

Para identificar las creencias de las estudiantes respecto a la realización del autoexamen de mama se empleó el cuestionario de actitudes hacia el autoexamen de mama basado en el Modelo de Creencias en Salud de Becker, elaborado y validado por Victoria Champion en 1984, adaptado al español y aplicado en Chile, por Schencke et al.(13), cuenta con 29 ítems divididos en cuatro subescalas: susceptibilidad percibida, gravedad percibida, beneficios percibidos y barreras percibidas, con valores aceptables de confiabilidad por Alfa de Cronbach que van de 0,681 a 0,826 .

Para identificar estilos de vida se identificó la Healthy Lifestyle Scale for University Students, desarrollada por Wang et al., en 2012 con 38 ítems, divididos en ocho subescalas: apoyo social, apreciación por la vida, conducta regular, conducta nutricional, conducta de ejercicio, conducta de riesgo, manejo del estrés y responsabilidad en salud; con valores aceptables de fiabilidad por Alfa de Cronbach de 0,695 a 0,786 , aplicando una escala Likert con valores de 1 al 5(14).

\section{Procedimiento}

La selección de las participantes se realizó a través de un muestreo polietápico, la primera etapa consistió en un muestreo estratificado por asignación proporcional, donde cada estrato representó un área disciplinar (ciencias de la salud, artes, ciencias biológicas-agropecuarias, económico-administrativas, humanidades y técnicas).

La segunda etapa consistió en un muestreo aleatorio por conglomerados, donde se obtuvo una licenciatura (conglomerado) por estrato, definiendo su tamaño por asignación proporcional (\%) de acuerdo al total de estudiantes inscritas en el periodo agostodiciembre 2016 de las licenciaturas seleccionadas de manera aleatoria (Nutrición, Fotografía, Biología, Gestión y dirección de negocios, Pedagogía e Ingeniería civil).

En la tercera etapa, se eligió un conjunto de materias por licenciatura, de acuerdo con su asignación por aula en orden alfabético o numérico en un horario determinado, posteriormente se accedió a cada aula cubriendo el total de las estudiantes presentes hasta completar la muestra.

\section{Análisis de datos}

Se realizó comparación de medias entre grupos independientes través la prueba t de Student, se aplicó correlación de Spearman para identificar relaciones entre los puntajes obtenidos en la escala de estilos de vida y la de creencias hacía la autoexploración de mama. Por último se realizaron análisis multivariados de regresión logística, donde las variables dependientes consideradas en los modelos fueron la realización del autoexamen de mama y la presencia de pareja, esta última se midió a través de una variable dicotómica, donde se respondía la presencia o ausencia de pareja, mientras las variables independientes se integraron por 
creencias y características sociodemográficas. Las pruebas estadísticas se realizaron a través del software de análisis estadístico SPSS versión 18.0, considerando en todos los casos como significativos valores de p inferiores a 0,05.

\section{Consideraciones éticas}

Los criterios éticos en esta investigación se sustentaron en los principios básicos plasmados en la Declaración de Helsinki de la Asociación Médica Mundial, Seúl, octubre de 2008, especialmente donde se menciona la privacidad de los sujetos de investigación y la confidencialidad de su información personal, durante el proceso de recolección de datos se entregó un consentimiento informado a las participantes, el cual debían firmar previamente. Se trata de un estudio "sin riesgo" para los participantes, en el que su participación se reguló con la firma del consentimiento informado en donde se aclaraba su carácter anónimo, voluntario y con posibilidades de declinar en cualquier momento(15) en apego a la normativa internacional y nacional vigentes.

\section{Resultados}

La media de edad fue de 20,32 años (DE $=2,67$ ), $84,5 \%$ de las estudiantes se encuentran inscritas entre $1^{\circ}$ y $5^{\circ}$ semestre. $50.34 \%$ de las estudiantes tienen pareja (noviazgo), $83,1 \%$ no trabaja, $80,9 \%$ son católicas, y $81 \%$ cuentan con seguridad social. $13,2 \%$ de las estudiantes tienen antecedentes heredofamiliares de cáncer de mama.

Del total de las estudiantes el $19,7 \%$ se realiza el autoexamen una vez al mes y 83,8\% conoce acerca del autoexamen de mama. Las fuentes de información más frecuentes son los medios de comunicación (29\%) y el personal de salud $(20.2 \%)$.

De las mujeres que se realizan la autoexploración mamaria, $73,8 \%$ lo hace en cualquier momento del ciclo menstrual y solo $11,5 \%$ lo realiza de manera correcta, es decir una vez al mes y en los 7 a 10 días después del inicio de la menstruación.

Tabla 1. Comparación de medias de edad, creencias en salud y estilos de vida de acuerdo con la realización del autoexamen de mama en las estudiantes universitarias

\begin{tabular}{|c|c|c|c|}
\hline Variables & $\begin{array}{c}\text { Realiza AEM } \\
(n=122) \\
\text { Media } \pm \text { DE }\end{array}$ & $\begin{array}{c}\text { No realiza AEM } \\
(n=162) \\
\text { Media } \pm \text { DE }\end{array}$ & $p$ \\
\hline $\begin{array}{l}\text { Edad } \\
\text { Creencias en salud }\end{array}$ & $21,1 \pm 3,5$ & $19,6 \pm 1,5$ & 0,000 \\
\hline Susceptibilidad & $11,5 \pm 2,9$ & $11,4 \pm 3,2$ & 0,774 \\
\hline Gravedad & $31,0 \pm 7,4$ & $31,0 \pm 7,5$ & 0,976 \\
\hline Beneficios & $21,3 \pm 3,0$ & $20,8 \pm 3,4$ & 0,185 \\
\hline Barreras & $15,7 \pm 5,0$ & $16,9 \pm 4,7$ & 0,048 \\
\hline \multicolumn{4}{|l|}{ Estilos de vida } \\
\hline Apoyo social & $22,4 \pm 3,2$ & $21,8 \pm 3,6$ & 0,125 \\
\hline Apreciación por la vida & $21,0 \pm 2,6$ & $20,1 \pm 2,8$ & 0,006 \\
\hline Conducta regular & $13,6 \pm 3,2$ & $12,6 \pm 3,3$ & 0,011 \\
\hline Conducta nutricional & $14,0 \pm 3,1$ & $13,0 \pm 3,3$ & 0,020 \\
\hline Conducta de ejercicio & $11,2 \pm 3,3$ & $10,6 \pm 3,7$ & 0,159 \\
\hline Conducta de riesgo & $9,3 \pm 2,3$ & $8,7 \pm 2,3$ & 0,018 \\
\hline Manejo del estrés & $17,2 \pm 3,4$ & $16,1 \pm 3,4$ & 0,012 \\
\hline Responsabilidad en salud & $24,8 \pm 2,6$ & $23,5 \pm 2,9$ & 0,000 \\
\hline
\end{tabular}

Comparación a través de la prueba t de student.

$\mathrm{DE}=$ Desviación estándar AEM= Autoexamen de mama. 
Tabla 2. Comparación de medias de creencias en salud y estilos de vida de acuerdo con la presencia o ausencia de pareja en las estudiantes universitarias

\begin{tabular}{|c|c|c|c|}
\hline \multirow[t]{2}{*}{ Variables } & Con pareja $(n=134)$ & Sin pareja $(n=132)$ & \multirow[t]{2}{*}{$p$} \\
\hline & Media \pm DE & Media \pm DE & \\
\hline \multicolumn{4}{|l|}{ Creencias en salud } \\
\hline Susceptibilidad & $11,2 \pm 3,1$ & $11,9 \pm 3,1$ & 0,072 \\
\hline Gravedad & $30,2 \pm 7,6$ & $31,4 \pm 7,1$ & 0,188 \\
\hline Beneficios & $21,5 \pm 2,8$ & $20,5 \pm 3,6$ & 0,016 \\
\hline Barreras & $15,6 \pm 4,8$ & $17,4 \pm 4,7$ & 0,002 \\
\hline \multicolumn{4}{|l|}{ Estilos de vida } \\
\hline Apoyo social & $22,0 \pm 3,5$ & $22.1 \pm 3.5$ & 0,713 \\
\hline Apreciación por la vida & $20,9 \pm 2,8$ & $20,2 \pm 2,7$ & 0,042 \\
\hline Conducta regular & $13,1 \pm 3,5$ & $12,9 \pm 3,1$ & 0,627 \\
\hline Conducta nutricional & $13,6 \pm 3,4$ & $13,1 \pm 3,0$ & 0,197 \\
\hline Conducta de ejercicio & $10,7 \pm 3,7$ & $10,9 \pm 3,3$ & 0,647 \\
\hline Conducta de riesgo & $9,0 \pm 2,5$ & $8,9 \pm 2,2$ & 0,919 \\
\hline Manejo del estrés & $16,7 \pm 3,6$ & $16,5 \pm 3,3$ & 0,582 \\
\hline Responsabilidad en salud & $24,1 \pm 2,8$ & $23,9 \pm 2,9$ & 0,640 \\
\hline
\end{tabular}

Comparación a través de la prueba t de student.

$\mathrm{DE}=$ Desviación estándar.

En la comparación de grupos, las estudiantes que realizan el autoexamen de mama mostraron menores barreras percibidas y mayor edad que aquellas que no lo realizan (Tabla 1).

En las estudiantes con pareja se evidenciaron mayores beneficios percibidos y menores barreras hacia el autoexamen de mama que aquellas sin pareja (Tabla 2 ).
En las correlaciones se identificó que a mayor susceptibilidad percibida existe menor conducta regular, nutricional y de ejercicio. De igual manera a mayor gravedad percibida menor apreciación por la vida y manejo del estrés y a mayores barreras percibidas menor apreciación por la vida, conducta nutricional, de ejercicio, manejo del estrés y responsabilidad en salud. Mientras que a mayores beneficios percibidos mayor apoyo social y conducta regular (Tabla 3).

Tabla 3. Correlaciones entre creencias respecto al autoexamen de mama y estilos de vida saludable en las estudiantes universitarias

\begin{tabular}{lllll}
\hline & Susceptibilidad & Gravedad & Beneficios & Barreras \\
\hline Apoyo social & .010 & -.033 & $.158^{* *}$ & -.010 \\
Apreciación por la vida & -.084 & $-.162^{* *}$ & .089 & $-.207^{* *}$ \\
Conducta regular & $-.126^{*}$ & .039 & $.121^{*}$ & -.097 \\
Conducta nutricional & $-.152^{*}$ & -.044 & .092 & $-.169^{* *}$ \\
Conducta de ejercicio & $-.203^{* *}$ & -.061 & .007 & $-.123^{*}$ \\
Manejo de estrés & -.096 & $-.151^{*}$ & -.050 & $-.118^{*}$ \\
Responsabilidad en salud & .017 & -.037 & .076 & $-.166^{* *}$ \\
\hline
\end{tabular}

${ }^{* *} \mathrm{p}<0.001{ }^{*} \mathrm{p}<0.05$ 
Tabla 4. Modelos multivariados para la realización del autoexamen de mama y variables asociadas a su práctica

\begin{tabular}{|c|c|c|c|c|c|c|}
\hline & \multicolumn{3}{|c|}{ Modelo crudo } & \multicolumn{3}{|c|}{ Modelo ajustado } \\
\hline & OR & IC $95 \%$ & $p$ (sig.) & OR & IC95\% & $p$ \\
\hline \multicolumn{7}{|c|}{ Modelo 1. Variable dependiente: realización del autoexamen de mama } \\
\hline \multicolumn{7}{|c|}{ Creencias en salud } \\
\hline Susceptibilidad & 1,02 & $(0,94-1,10)$ & 0,609 & - & - & 0,497 \\
\hline Gravedad & 1,00 & $(0,97-1,04)$ & 0,644 & - & - & 0,493 \\
\hline Beneficios & 1,03 & $(0,96-1,12)$ & 0,318 & - & - & 0,282 \\
\hline Barreras & 0,94 & $(0,90-1,00)$ & 0,052 & 0,95 & $(0,90-0,99)$ & 0,049 \\
\hline
\end{tabular}

Modelo 2. Variable dependiente: realización del autoexamen de mama

Estilos de vida

\begin{tabular}{|c|c|c|c|c|c|c|}
\hline Apoyo social & 0,98 & $(0,90-1,06)$ & 0,66 & - & - & 0,931 \\
\hline Apreciación por la vida & 1,02 & $(0,91-1,15)$ & 0,695 & - & - & 0,301 \\
\hline Conducta regular & 1,07 & $(0,98-1,18)$ & 0,113 & 1,10 & $(1,01-1,20)$ & 0,018 \\
\hline Conducta nutricional & 1,01 & $(0,92-1,12)$ & 0,72 & - & - & 0,518 \\
\hline Conducta de ejercicio & 1,00 & $(0,92-1.08)$ & 0,986 & - & - & 0,799 \\
\hline $\begin{array}{l}\text { Responsabilidad } \\
\text { en salud }\end{array}$ & 1,10 & $(0,99-1,23)$ & 0,062 & 1,13 & $(1,02-1,25)$ & 0,015 \\
\hline Conducta de riesgo & 1,18 & $(1,05-1,32)$ & 0,004 & 1,17 & $(1,05-1,30)$ & 0,003 \\
\hline Manejo del estrés & 1,05 & $(0,96-1,16)$ & 0,259 & - & - & 0,142 \\
\hline Edad & 1,40 & $(1,21-1,66)$ & 0,000 & 1,40 & $(1,21-1,65)$ & 0,000 \\
\hline
\end{tabular}

Modelo 3. Variable dependiente: Presencia de pareja

Creencias en salud

\begin{tabular}{|c|c|c|c|c|c|c|}
\hline Susceptibilidad & 0,94 & $(0,86-1,02)$ & 0,164 & - & - & 0,143 \\
\hline Gravedad & 0,99 & $(0,96-1,03)$ & 0,899 & - & - & 0,641 \\
\hline Beneficios & 1,08 & $(1,00-1,17)$ & 0,039 & 1,08 & $(1,00-1,17)$ & 0,046 \\
\hline Barreras & 0,93 & $(0,88-0,98)$ & 0,019 & 0,93 & $(0,88-0,97)$ & 0,006 \\
\hline
\end{tabular}

En los análisis multivariados se encontró que menores barreras percibidas y mayor edad son factores que predisponen a la realización del autoexamen de mama en las estudiantes universitarias, mientras que tener pareja se asocia de manera significativa con menores barreras y mayores beneficios percibidos hacia el autoexamen (Tabla 4).

\section{Discusión}

El autoexamen de mama, es una herramienta que permite a la mujer tener conocimiento de su propio cuerpo, familiarizarse con sus mamas y poder identificar cualquier anormalidad(16), siendo el vínculo para la demanda de atención médica oportuna. Algunos estudios señalan que su práctica se encuentra por debajo del $30 \%{ }^{8-}$ 
10,17), lo que refleja un déficit de autocuidado en las prácticas de detección oportuna del cáncer de mama y en la demanda de la atención.

En este estudio, la proporción de estudiantes que realiza el autoexamen de manera mensual fue de $19,7 \%$ similar a lo encontrado en Malasia(9), siendo un valor superior a lo reportado en Buea Camerún(18), Pamplona, Colombia y Caracas, Venezuela(19); y con un valor inferior a lo obtenido en Ordu, Turquía(17), Riad Arabia Saudita(20); Emiratos Árabes(10), Shah Alam, Malasia(12) y Pamplona, Colombia(11). De lo anterior, se puede considerar que existe tendencia a la no realización del autoexamen en las estudiantes universitarias, tanto en países de Oriente como en América Latina.

En esta investigación 83,8\% de las estudiantes conocen sobre el autoexamen de mama, similar a lo encontrado en Kaffa, Etiopía(8), en Lima Perú(21,22), donde se hace evidente la separación que existe entre el conocimiento que pueda poseer un individuo sobre un riesgo particular y su conducta concreta, pues sus comportamientos no corresponden con dicho conocimiento(23); lo que lleva a repensar la sobreestimación habitual de la transmisión de conocimientos para formar cambios en la conducta en salud.

La edad presentó asociación significativa con la realización del autoexamen, lo que coincide con Akhtari-Zavare, et al., en Klang Valley, Malasia( ${ }^{(9)}$, Abolfotouh, et al., en Arabia Saudita(20); y AlDubai, et al., en Shah Alam, Malasia(12). El incremento de la edad es uno de los factores de riesgo para desarrollar cáncer de mama en las mujeres(16), por lo que podría esperarse que aquellas mujeres de menor edad tengan menor predisposición a realizar el autoexamen de mama, dado que se sienten menos susceptibles a padecer cáncer de mama.

La asociación identificada entre las barreras percibidas hacia el autoexamen y la realización del mismo, coincide con lo obtenido por AkhtariZavare $\mathrm{M}$, et al.(9), y Abolfotouh $\mathrm{M}$, et al., en Arabia Saudita(20), congruente con la teoría del Modelo de Creencias en Salud de Becker, donde las barreras percibidas hacia la conducta en salud obstaculizan su realización(6,7,24). En la medida en que las mujeres identifican barreras al parecer omiten la práctica de esta conducta de autocuidado.

Por otra parte, esta investigación muestra la relación entre la presencia de pareja que en este caso se trata de noviazgo y la disminución de barreras percibidas hacia el autoexamen de mama en las estudiantes universitarias, reforzando lo encontrado por Al-Dubai, et al. (12), y Al-Sharbatti, et al(10), donde mostraron que las mujeres casadas tienen mayor predisposición a la práctica del autoexamen que aquellas solteras. Aun cuando se había identificado que el estado civil predispone la realización del autoexamen, en esta investigación se encontró que más allá del estado civil el hecho de tener una pareja favorece la disminución de barreras hacia la realización del autoexamen, lo cual es consistente con lo encontrado en otros estudios, donde la presencia de pareja, se asocia con el autocuidado suficiente en personas con enfermedades cardíacas(25), es fuente de apoyo para afrontar enfermedades crónicas, como diabetes mellitus y favorecer el control metabólico $(p<0,05)^{(26,27)}$, además contribuyen con la adherencia al tratamiento farmacológico(28). Por su parte Gallardo-Peralta L., et al., destacan la importancia del cónyuge como fuente de apoyo en personas con depresión(29).

Varela, et al., ya habían identificado asociación entre la relación de pareja y el cumplimiento de las pautas recomendadas al paciente que incluyen alimentación, ejercicio físico y disminución de consumo de tabaco(30). Haciendo evidente que el apoyo social se relaciona con la adopción de conductas en salud y prácticas de autocuidado. Si se tiene presente que el apoyo social se refiere a la existencia o disponibilidad de personas en quienes se puede confiar en momentos de adversidad(28), las parejas pueden cumplir perfectamente con esta función resultando en un factor protector de la salud. La adopción de las prácticas de autocuidado, como el autoexamen, es más favorable en aquellas estudiantes que mantienen redes de apoyo estables. La realización del autoexamen involucra 
diversos elementos del individuo como percepciones, relaciones interpersonales, el contexto cultural y social, mismos que deben ser considerados por los salubristas y profesionales de la salud al momento de intervenir en la población.

La principal fortaleza de este trabajo es el abordaje de variables poco consideradas en la literatura como son las relaciones de pareja en mujeres solteras (noviazgo) y la influencia que puede tener en conductas de autocuidado como es la autoexploración mamaria. Sin embargo, una de las grandes limitaciones del trabajo es su diseño transversal que no permite determinar si las relaciones encontradas son causales por lo cual no puede afirmarse que las relaciones de parejas sean quienes determinan menores barreras, pudiera ser que jóvenes con mayores beneficios percibidos y menores barreras percibidas hacia el autoexamen, tiendan a entablar con mayor frecuencia relaciones de noviazgo. Por lo anterior, en estudios posteriores se sugiere la realización de estudios longitudinales para determinar causalidad.

\section{Conclusiones}

Existe una separación entre el conocimiento y la práctica del autoexamen. Las estudiantes tienen conocimiento acerca del autoexamen de mama, sin embargo, no es suficiente para motivar su práctica, pues en este estudio se ha demostrado que interfieren las creencias en salud y las relaciones interpersonales.

En mujeres jóvenes solteras, el tener relaciones de pareja se relaciona con menores barreras percibidas y mayores beneficios percibidos hacia el autoexamen de mama. A su vez las barreras percibidas parecen ser el principal factor que evita la realización del autoexamen, por lo que el apoyo de la pareja pudiera estar jugando un papel importante.

\section{Conflicto de intereses}

No existen conflictos de intereses.

\section{Referencias}

1. Martínez O, Uribe P, Hernández M. Políticas públicas para la detección del cáncer de mama en México. Salud Pública Mex [Internet]. 2009;51(1):s350-60. Available from:

http://www.redalyc.org/pdf/106/10616164028.pdf

2. Arias M, González C, Alcaraz C, Carvajal MG. Efectos psicosociales en la mujer con mastectomía. Políticas Soc Sect. 2016;3(3).

3. Secretaría de Salud. Programa de Acción Específico Prevención y Control del Cáncer de la Mujer [Internet]. México: Secretaria de Salud; 2018. Available from: http://www.spps.gob.mx/programas-accion20132018.html

4. Torre LA, Bray F, Siegel RL, Ferlay J, Lortet-tieulent J, Jemal A. Global Cancer Statistics, 2012. CA a cancer J Clin [Internet]. 2015;65(2):87-108. Available from: http://onlinelibrary.wiley.com/doi/10.3322/caac.2126 2/abstract

5. Organización Mundial de la Salud. Cáncer de mama: prevención y control [Internet]. Ginebra: OMS; 2014 [cited 2015 Oct 10]. Available from: http://www.who.int/topics/cancer/breastcancer/es/

6. Moreno E, Roales-Nieto J. El Modelo de Creencias de Salud : Revisión Teórica , Consideración ... Int J Psychol Psychol Ther. 2003;3(1):91-109.

7. Cabrera A, Tascón G, Lucumí C. Creencias en salud: historia, constructos. Rev Fac Nac Salud Pública. 2001;19(1):91-101.

8. Birhane N, Mamo A, Girma E, Asfaw S. Predictors of breast self - examination among female teachers in Ethiopia using health belief model. Arch Public Heal [Internet]. 2015;73(1):39. Available from: http://www.archpublichealth.com/content/73/1/39

9. Akhtari-Zavare M, Juni M, Ismail I, Said S, Latiff L. Health Beliefs and Breast Self-Examination among Undergraduate Female Students in Public Universities in. Asian Pacific J Cancer Prev. 2015;16:4019-23.

10. Al-Sharbatti S, Shaikh R, Mathew E, Al-Biate M. Breast self-examination practice and breast cancer risk perception among female university students in Ajman. Asian Pacific J Cancer Prev. 2013;14(8):4919-23.

11. Martínez-Torres J, Pabón-Rozo C, Quintero-Contreras N, Soto Galván J, López-Mayorga R, Rojas-Tinico Y, et al. Barreras asociadas a la realización del autoexamen de seno en mujeres de 18 a 50 años de edad : un estudio descriptivo. Nutr Hosp. 2015;32(4):1664-9.

12. Al-Dubai S, Ganasegeran K, Alabsi A, Manaf M, Ijaz S, Kassim S. Exploration of barriers to breast-self examination among urban women in Shah Alam, Malaysia: A cross sectional study. Asian Pacific J Cancer Prev. 2012;13(4):1627-32.

13. Schencke M, Espinoza S, Muñoz N, Messing H. Actitud y conducta frente al autoexamen de mama entre profesionales de salud en Chile. Pan Am J Public Heal [Internet]. 1993;114(4):317-325. Available from: http://iris.paho.org/xmlui/handle/123456789/16272

14. Wang D, Xing X, Wu X. The Healthy Lifestyle Scale for University Students: development and psychometric testing. Aust J Prim Health [Internet]. 2012;18:339-45. 
Available

from:

https://www.ncbi.nlm.nih.gov/pubmed/22950906?rep ort=docsum\&format=text

15. Comisión Nacional de Bioética. Guía nacional para la integración y el funcionamiento de los Comités de Ética en Investigación. México: Secretaría de Salud/Comisión Nacional de Bioética; 2016. 1-66 p.

16. Secretaría de Salud. Norma Oficial Mexicana NOM-041SSA2-2011. Mexico: Secretaría de Salud; 2011.

17. Erbil N, Bolukbas N. Health beliefs and breast selfexamination among female university nursing students in Turkey. Asian Pacific J Cancer Prev. 2014;15(16):6525-9.

18. Nade P, Nguedia J, Kwenti E, Njunda A. Knowledge, attitude and practice of breast self-examination among female undergraduate students in the University of Buea. BMC Res Notes [Internet]. 2015;8:43. Available from:

https://www.ncbi.nlm.nih.gov/pmc/articles/PMC4414 436/pdf/13104_2015_Article_1004.pdf

19. Pengpid S, Peltzer K. Knowledge, attitude and practice of breast self-examination among female university students from 24 low, middle income and emerging economy countries. Asian Pac J Cancer Prev [Internet]. 2014;15(20):8637-40. Available from: http://www.ncbi.nlm.nih.gov/pubmed/25374181

20. Abolfotouh M, BaniMustafa A, Mahfouz A, Al-Assiri M, Al-Juhani A, Alaskar A. Using the health belief model to predict breast self examination among Saudi women. BMC Public Health [Internet]. 2015;15(1):1163. Available from: http://www.biomedcentral.com/14712458/15/1163

21. Carrillo R, Espinoza M, Osada J, Ávilez J. Nivel de conocimiento y frecuencia de autoexamen de mama en alumnos de los primeros años de la carrera de Medicina. Rev Med Hered [Internet]. 2015;26:209-16. Available from: http://www.upch.edu.pe/vrinve/dugic/revistas/index. php/RMH/article/view/2699/2575

22. Mazzini C. Knowledge and Practice of the Breast SelfExam on Students from a Public University in Lima. Arch Can Res. 2016;4:1-4.

23. De Roux GI. La prevención de comportamientos de riesgo y la promoción de estilos de vida saludable en el desarrollo de la salud. Educ Med Salud [Internet]. 1994;28(2):223-33. Available from: http://www.sld.cu/galerias/pdf/sitios/gericuba/preve ncion_y_promocion.pdf

24. Glanz K, Rimer B, Viswanath K. The health belief model. Health behavior and health education: Theory, research, and practice. 4th ed. Jossey- Bass; 2008. 4565pp p.

25. Gázquez R, Ángeles MDL, Holguín A, Acevedo S, Andrea Y. Capacidad de agencia de autocuidado y factores relacionados con la agencia en personas con insuficiencia cardíaca de la ciudad de Medellín ( Colombia ) Self-care agency ability and factors related to the agency in people with heart failure of. Enfermería Glob [Internet]. 2013;183-95. Available from: http://scielo.isciii.es/scielo.php?pid=S1695-

61412013000200009\&script=sci_arttext
26. Arteaga Noriega A, Cogollo Jiménez R, Muñoz Monterroza D. Apoyo social y control metabólico en la diabetes mellitus tipo 2 . Rev Cuid [Internet]. 2017;8(2):1668. Available from: https://www.revistacuidarte.org/index.php/cuidarte/ article/view/405

27. Herrera A, Andrade Y, Hernández O, Manrique J, Faria $\mathrm{K}$, Machado M. Personas con diabetes mellitus tipo $2 \mathrm{y}$ su capacidad de agencia de autocuidado. Av en Enfermería [Internet]. 2012;30(2):39-46. Available from:

http://www.scielo.org.co/pdf/aven/v30n2/v30n2a03. pdf

28. Canales S, Barra E. Autoeficacia, apoyo social y adherencia al tratamiento en adultos con diabetes mellitus tipo. Psicol y Salud. 2014;24(2):167-73.

29. Gallardo-Peralta LP, Sánchez-Moreno E, Arias-Astray A, López-de-Roda AB. Elementos estructurales de la red social, fuentes de apoyo funcional, reciprocidad, apoyo comunitario y depresión en personas mayores en Chile. An Psicol. 2015;31(3):1018-29.

30. Varela Montero I, Barrón López de Roda A. La influencia de la pareja en el ajuste a la enfermedad cardiaca. Acta Investig Psicológica [Internet]. 2016;6(2):2459-68. Available from: http://linkinghub.elsevier.com/retrieve/pii/S2007471 916300217 